\title{
A Method for New Energy Electric Vehicle Charging Hole Detection and Location Based on Machine Vision
}

\author{
Hui Zhang ${ }^{1,2, a}$, Xiating Jin ${ }^{1, b}$ \\ ${ }^{1}$ College of Electrical and Information Engineering, Changsha University of Science and \\ Technology, Changsha 410012, China; \\ ${ }^{2}$ College of Electrical and Information Engineering, Hunan University, Changsha 410082, China. \\ azhanghuihby@126.com, ${ }^{\mathrm{b}} 76788651 @ q q . c o m$
}

Keywords: new energy electric vehicle charging, detection and location to charging hole, machine vision, $\mathrm{HSI}$ color model, morphology.

\begin{abstract}
A new method based on machine vision is designed for electric vehicle charging hole detection and location in order to solve the low efficiency, space limitations or leakage risk in artificial charging operation for electric vehicle and to realize the automatic charging based on robot. The method enable to efficiently and accurately extract valuable characteristics of the charging hole from a charging socket image. Aim at the problem that strong electromagnetic automobile charging system will bring salt and pepper noise to image signal, the paper firstly adopts the classical median filtering for image noise cancellation. The charging socket image shows complicated background, uneven brightness, strong reflective and few goal characteristic, making the goal segmentation extremely difficult when employing the common method with fixed or adaptive threshold. Therefore, a two-stage image segmentation method based on HSI color model is proposed in paper to extract the characteristics of the charging hole target with sub-pixel precision. The image segmentation method involves threshold segmentation in the Hue component of original image, morphological operation and edge detection based on Canny operator. Meanwhile, It also reduces the influence of problem above due to the operation in Hue component. In this paper, it is based on vision platform HALCON and experiment result shows that the method enable to meet the requirements where location accuracy with sub-pixel precision and detection.
\end{abstract}

\section{Introduction}

Automobile industry, as the national economic lifeline, driving a large upstream and downstream industry chain, is the national major strategic pillar industry. New energy automobile as a bright spot of modern car industry, is the important engine to promote sustainable economic and social development. By the end of 2015, there are a total of 583200 new energy vehicles in China, with the growth rate of nearly $170 \%$, indicating the automobile industry is in the trend of an explosive development [1].

Different kinds of new energy electric vehicles, such as Electric Vehicle (EV), Extended Range Electric Vehicle (EREV) and Hybrid Electrical Vehicle (HEV), are equipped with energy storage system which needs to recharge periodically $[2,3]$. Usually, linking the energy storage systems with a power supply, the AC power lines for example, can charge for it.

Although it is beneficial to recharge the energy storage system before or after a vehicle is in use, the current system requires vehicle driver to link the power-supply line with vehicle artificially and manually. The charging way exists many disadvantage factors, restricting the driver himself and the development of the electric car industry, such as artificial operation with charging guns leads to low efficiency and/or miss the best charging time, vehicle driver is not convenient for outdoor operation in bad weather conditions, power lines may appear leakage fault so that there are potential safety hazards. Therefore, it urgently needs a manpower saving, time saving, safe and reliable automatic charging method, in order to realize the charging process efficient, accurate and safe. For this purpose, this paper presents a charging method based on machine vision for new energy electric vehicle. 


\section{Image Detection Method}

The process of image processing described in this article is as shown in figure 1, there are many steps involved, such as image filtering, threshold segmentation, morphology processing, edge detection with accuracy of sub-pixel, robustness and feature extraction, etc.

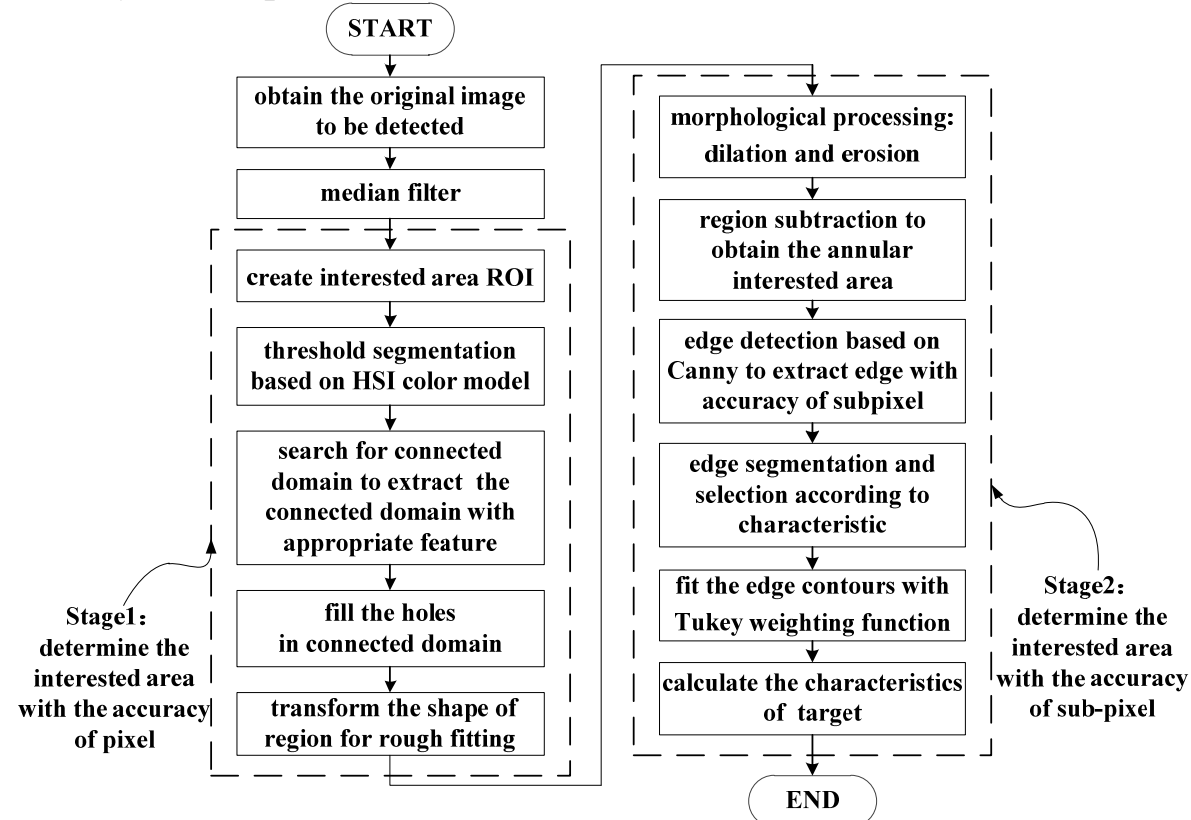

Fig. 1 The flow chart of image processing for charging hole detection and location

\subsection{Image Filtering}

In the automobile charging station system, there is a very strong complex electromagnetic environment, which can be roughly divided into three classes, namely vehicle electromagnetic source, charging pile electromagnetic source and natural sources. Electric vehicles have high-pressure and high-power power electronic device and the drive motor, at the same time, compared with the traditional cars increase more high voltage wire and CAN communication wiring harness, would have serious electromagnetic interference; Many wire, coil and electronic components of the AC/DC charging pile have different sizes of inductance and capacitance loop, when an electrical spark in work, can produce high-frequency oscillation and firing into the air in the form of electromagnetic waves. Automobile charging operation, therefore, is a complex process interfered by multiple electromagnetic system, bringing to the image signal collection, transmission and transformation many kinds of noise, among them the salt and pepper noise is typical. The noise will cause the loss of image quality and difficult to image analysis and recognition, so it is very important for the original image filtering.

Usually, linear smoothing filtering and nonlinear smoothing filter are used for image filtering. Because the salt and pepper noise in image and background is not related, which belongs to the high frequency components, and linear filter are mostly low pass, so we consider using the nonlinear filter: median filtering algorithm is a typical nonlinear filtering algorithm, the principle of which is that it can sort image pixels in field according to gray scale sorted, and then select the intermediate values as the output pixels to remove noise and enhance the effect of the image $[4,5]$. Median filtering is defined as:

$$
g(x, y)=\operatorname{Med}\{f(x-k, y-l), k, l<W\}
$$

where $g$ is the image after median filtering, $f$ is the original image, $W$ is the size of the filter window, Med is the value.

\subsection{Threshold Segmentation Based on HSI Color Model}

The essence of threshold segmentation is to divide the collection of pixels in accordance with the gray scale, and then each subset form a mundane corresponding area, each of which have consistent attributes and adjacent areas do not have this same attributes. 
Direct threshold segmentation, often is not applicable to the separation of complex scene, so we consider the color features of image. Different color model in different color space will express the effect in a big gap, which directly affects the understanding of the image. Therefore, selecting the appropriate color space to analyze can improve the effect of recognition. In this study, based on HSI color model $[6,7]$, we use two modified two-dimensional image threshold segmentation method.

1) Maximum between-class variance method

The maximum between-class variance method is put forward by Otsu in 1978, which is a kind of adaptive threshold-determining method. It gets minimum interclass variance between the two groups of pixel values to determine the threshold. If we define the histogram function as a probability function $\mathrm{P}$, of which the $\mathrm{P}(0), \mathrm{P}(1), \ldots \mathrm{P}(\mathrm{I})$ is the probability of histogram whose grey value is $0,1 \ldots \mathrm{I}$, OTSU about the best threshold value is defined as the interclass variance weighted and minimum threshold, among them the rights are referred to as the probability of each group. The simplified calculation formula of the algorithm is as follows:

$$
\sigma_{T}^{2}=\omega_{a}\left(\mu_{a}-\mu\right)^{2}+\omega_{b}\left(\mu_{b}-\mu\right)^{2}
$$

where $\sigma_{T}^{2}$ is the maximum variance between two classes, $\omega_{a}$ is the probability of class $A, \mu_{a}$ is the average gray level of class $A, \omega_{b}$ is the probability of class $B, \mu_{b}$ is the average gray level of class $B$, $\mu$ is the average gray level images. The image is divided into part $A$ and part $B$ by value $T$, making the total variance $\sigma_{T}^{2}$ get the maximum value of $T$, namely the best segmentation threshold.

2) Calculate maximum frequency grey value of the histogram

The analysis to the hue of charging hole image is as follows: due to the special metal in hole core, together with certain reflective effect, it makes the region on the original image in a certain range of the same color. From the perspective of human eyes, the region shows obvious golden yellow or bright/or partial dark, which belongs to warm hue. However, the edge of core hole, thought to be sage green plastic, belongs to dark or cold hue, making the hue of this region is greatly different from core hole's. Therefore, using this feature, we can separate charging hole core from background image in hue component. The specific steps are: (1) calculate the absolute gray value histogram and relative gray value histogram of image; (2) find out the grey value with peak frequency, namely maximum; (3) use a nearby value of maximum as the threshold in the global threshold segmentation, as shown below:

$$
f(x, y)=\left\{\begin{array}{cc}
255 & g(x, y) \geq M \\
0 & g(x, y)<M
\end{array}\right.
$$

where $g$ is the image before segmentation, $f$ is the image after segmentation, $M$ is a nearby value of maximum.

\subsection{Edge Detection Based on Morphology and Canny Operator}

1) Obtain annular region by mathematical morphology

The first step is to dilate the interested area above to get a circular area, written as C1. Next we erode the interested area above to get another circular area, written as C2. Finally, we subtract the two areas to obtain an annular region, namely to subtract $\mathrm{C} 1$ from $\mathrm{C} 2$. The dilation and erosion are two kinds of dual basic operation in mathematical morphology, which are defined as:

$$
\begin{aligned}
& A \oplus B=\{x \mid B(x) \cap A \neq \varnothing\} \\
& A \odot B=\{x \mid B(x) \subset A\}
\end{aligned}
$$

(5)

where $A$ and $B$ are mathematical sets. (4) means $A$ is dilated by $B$. (5) means $A$ is eroded by $B$.

2) Sub-pixel edge detection by Canny operator

The process of sub-pixel edge detection based on Canny operator are as follows:

First step is Gaussian filtering, which can weight one dimensional Gaussian kernel (6), also can have convolution on two-dimensional Gaussian kernel (7) to realize. 


$$
\begin{aligned}
& K=\frac{1}{\sqrt{2 \pi} \sigma} e^{-\frac{x^{2}}{2 s^{2}}} \\
& K=\frac{1}{2 \pi \sigma^{2}} e^{-\frac{x^{2}+y^{2}}{2 \sigma^{2}}}
\end{aligned}
$$

Followed by the first order partial derivative of the finite difference can be used to calculate the gradient magnitude and direction angle. The first order partial derivative matrix $S_{x}, S_{y}$ of $x$ orientation and $y$ orientation are:

$$
S_{x}=\left[\begin{array}{ll}
-1 & 1 \\
-1 & 1
\end{array}\right], S_{y}=\left[\begin{array}{cc}
1 & 1 \\
-1 & -1
\end{array}\right]
$$

Next, the expressions for gradient amplitude and gradient direction angle are:

$$
\begin{aligned}
& P[i, j]=(f[i, j+1]-f[i, j]+f[i+1, j+1]-f[i+1, j]) / 2 \\
& Q[i, j]=(f[i, j]-f[i+1, j]+f[i, j+1]-f[i+1, j+1]) / 2 \\
& M[i, j]=\sqrt{P[i, j]^{2}+Q[i, j]^{2}} \\
& \theta[i, j]=\arctan (Q[i, j] / P[i, j])
\end{aligned}
$$

where $P[i, j]$ and $Q[i, j]$ are the gradient of each position on image, $M[i, j]$ is the gradient amplitude and $\theta[i, j]$ is the gradient direction angle.

Finally, non-maximum suppression and dual-threshold edge detection are performed [8].

\subsection{Edge Fitting Robustly for Characteristic Calculation}

In the traditional least squares principle: it requires that the distance sum of each point to the fitting straight line accesses minimum. Least squares for outliers far away from the straight line lack a good robustness, when using the least squares fitting a straight line, the points far away from straight line will be a big weight in the process of computing, results in an inaccurate line. So we need to think a method to cancel outlier or give it a small weight, in order to reduce the impact of these points.

As for the processing of weight value, there are different methods. This article adopts the method of Tukey weight function, which is a kind of robust method increasing the weight and reducing the effects of outliers.

$$
\omega(\delta)=\left\{\begin{array}{c}
{\left[1-\left(\frac{\delta}{\tau}\right)^{2}\right],|\delta| \leq \tau} \\
\frac{\tau}{|\delta|},|\delta|>\tau
\end{array}\right.
$$

where $\omega(\delta)$ is weighting function, $\delta$ is distance between point and straight line, $\tau$ is distance threshold.

When distance from point to straight line is greater than the threshold, the weighting function is equal to the reciprocal of the distance multiplied by the threshold, so that the farther the distance, the smaller the value; When distance is less than or equal to threshold, weight divided by the threshold value is given by the distance to the square of the difference square and 1, so that the smaller the distance, the greater the weight value.

After fitting the edge, there are some special information [9]. The extraction of image features, in this paper, we consider particularly center coordinate and area feature of each charging hole:

(1) Calculate the target center $O\left(x_{0}, y_{0}\right)$, namely the circle of charging hole. As shown below:

$$
O\left(x_{0}, y_{0}\right)=\left(\sum_{i=1}^{M} x_{i} / M, \sum_{j=1}^{M} y_{j} / M,\right), \forall\left(x_{i}, y_{j}\right) \in S
$$

where $M$ is the sum of pixel points, $S$ is target area.

(2) Calculate the characteristics of target area, mainly is through Green's Theorem to calculate 
area and then discretize, the essence of which is the number of pixels in target area $[10,11]$.

\section{The Experiment and Analysis}

The followings are based on vision software HALCON as the platform in different operation environments [12], such as the light source, shooting distance. It is through the algorithm above to detect and locate the charging hole, then compare with the experiment results and analyze.

\subsection{Image Acquisition}

This study adopts -IDS UI-6104SE-C/M CCD and TOSHIBA PORTEGE M909 computer to build machine vision detection system. Camera imaging plane should be parallel to the object to be tested. If the camera could not meet this requirement, or have bigger radial distortion, we should have camera calibration to adjust image. Now we read the charging hole original image shot by camera through the operator read_image( ).

\subsection{Image Filtering}

In order to remove the salt and pepper noise in image, usually we adopt median filtering of $3 * 3$ template or $7 * 7$ template. Here the operator used is median_image( ).

The experiments above are conducted in two conditions, that is $200 \mathrm{~mm}$ shooting distance, bright light and $600 \mathrm{~mm}$ shooting distance, bright light. Through the experimental results it can be seen that the median filtering for isolated eliminate noise well and make less fuzzy. But for some outlet images shot from far distance, the charging hole seems little, at this time if you use a larger template (like $7 * 7$ ) for median filtering, charging hole will be mistaken as noise and will be eliminated wrongly. Therefore, if there is noise, median filtering of $3 * 3$ template works best.

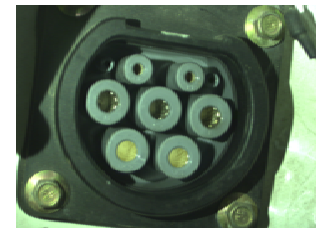

(a) $200 \mathrm{~mm}$, bright

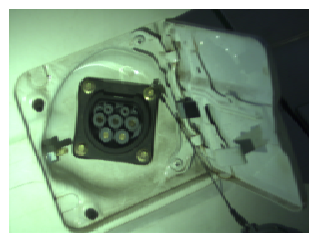

(c) $600 \mathrm{~mm}$, bright

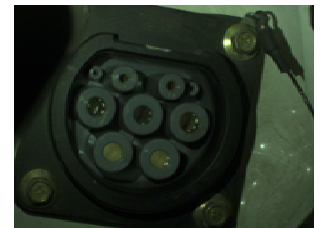

(b) $200 \mathrm{~mm}$, dark

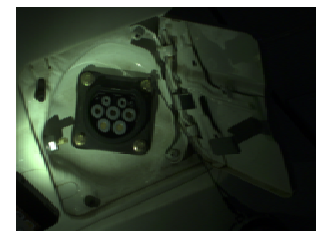

(d) $600 \mathrm{~mm}$, dark

Fig. 2 The original image
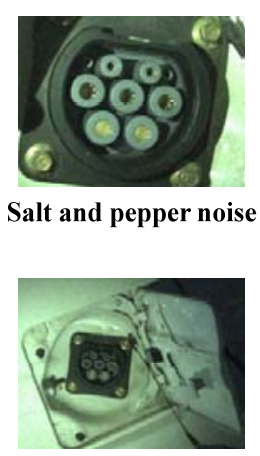

Salt and pepper noise

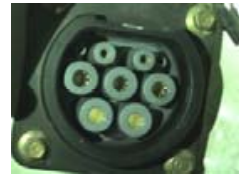

$3 * 3$ template (a) $200 \mathrm{~mm}$, bright

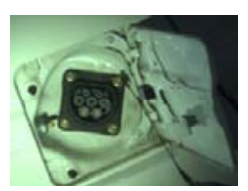

$3 * 3$ template (b) $200 \mathrm{~mm}$, dark

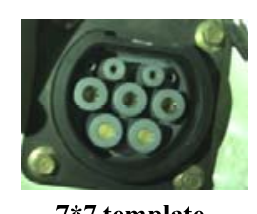

$7 * 7$ template

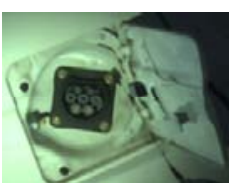

$7 * 7$ template

Fig.3 De-noised image

\subsection{Threshold Segmentation Based on HSI Color Model}

The purpose of threshold segmentation, essentially is to extract binary image from the charging hole image. However, the edge region of the charging hole core arouse controversy a lot. For this, HSI color model has made the very good solve, as shown in the analysis to the hue of charging hole image from the above section Calculate maximum frequency grey value of the histogram.

In the hue component of original image, charging hole core could be separated from the background image. Firstly, we transform the original image to HSI color space by the operators decompose3( ) and trans_from_rgb( ), to achieve the hue component. Next, based on HSI we adopt two kinds of two-dimensional image gray-level threshold segmentation method, namely maximum between-class variance method (method 1) and calculating maximum frequency grey value of the histogram (method 2). For the method 1, we could adopt the operator binary_threshold( ) to realize. For the method 2, its specific steps are: (1) calculate the absolute gray value histogram and relative gray value histogram of image by operator gray_histo( ); (2) find out the grey value with peak frequency, namely maximum, by operator PeakGray := sort_index(AbsoluteHisto)[255]; (3) use a nearby value of maximum as the threshold in the global threshold segmentation by operator threshold( ). 


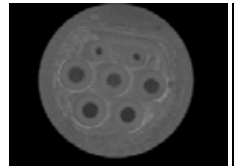

Hue component (a) $200 \mathrm{~mm}$, bright

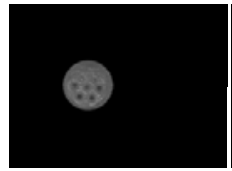

Hue component

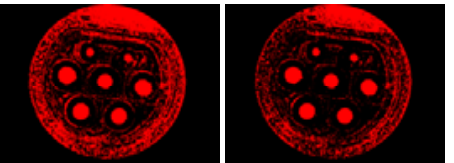

method 2

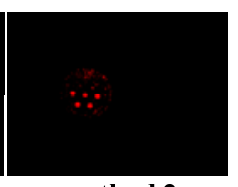

method 2 method 1 (c) $600 \mathrm{~mm}$, bright

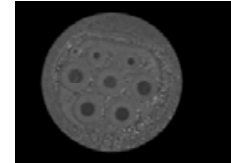

Hue component

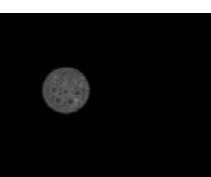

Hue component

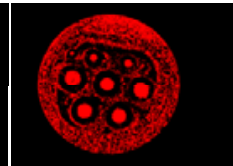

method 1 (b) $200 \mathrm{~mm}$, dark

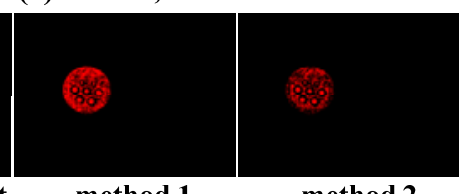

method 1 (d) $600 \mathrm{~mm}$, dark

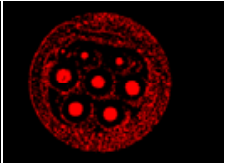

method 2

method 2

Fig.4 Binary image

Through the experimental results above, it can be seen that for the images shot close, two methods are applicable and have good segmentation effect; But for the images taken at a distance, if under the condition of bright light, method 1 is barely to realize segmentation, but under the dark condition, the processed images appear a large number of burr, making segmentation error is great. Method 2, therefore, is suitable for the broad conditions (shooting distance, illumination brightness), and the method 1 is only applicable to close shooting distance and/or the bright light.

\subsection{Preliminary Location of Charging Hole}

The first step is to take connected domain operation on the binary image above by the operator connection( ). Next is to extract the connected domain according to the characters of roundness and area by the operator select_shape( ), usually the roundness take $0.8 \sim 1$ and the area depends on actual situation. Then is to fill the hole to obtain not-empty connected domain by the operator fill_up( ) and to fit the contour according to convex hull by the operator shape trans( ). And the last step is to merger connected domain by the operator union1( ).

So far the interested charging hole region has been segmented from background image. Because of some little light, texture and lettering, however, simple binary operation can not meet the high precision location of charging hole. Therefore, it is necessary to locate precisely in order to ensure the accuracy of center central position.

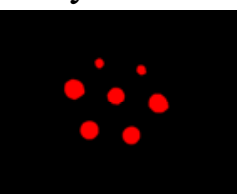

(a) $200 \mathrm{~mm}$, bright

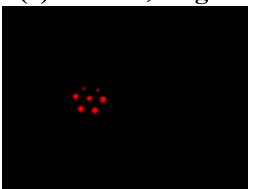

(c) $600 \mathrm{~mm}$, bright

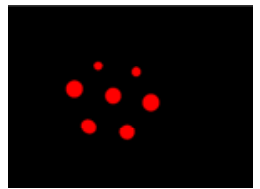

(b) $200 \mathrm{~mm}$, dark

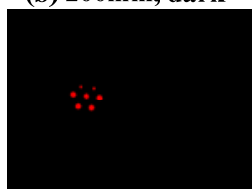

(d) $600 \mathrm{~mm}$, dark

Fig. 5 The coarse interested region
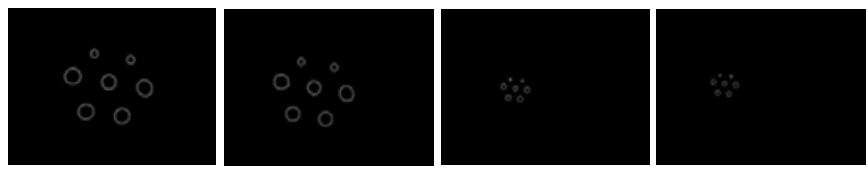

(a) $200 \mathrm{~mm}$, bright (b) $200 \mathrm{~mm}$, dark (c) $600 \mathrm{~mm}$, bright (d) $600 \mathrm{~mm}$, dark Fig.6 The interested annular region
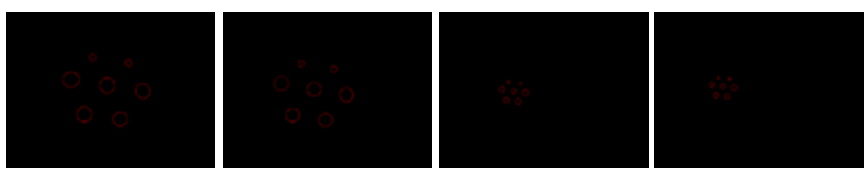

(a) $200 \mathrm{~mm}$, bright (b) $200 \mathrm{~mm}$, dark (c) $600 \mathrm{~mm}$, bright (d) $600 \mathrm{~mm}$, dark

Fig.7 Edge with the subpixel precision

\subsection{Obtainment of Annular Region by Mathematical Morphology}

The first step is to dilate and erode the interested region above to get two circular areas $\mathrm{C} 1$ and C2, where we realize it by the operators dilation_circle( ) and erosion_circle( ). Next is to subtract the two areas by the operator difference( ) to obtain an annular region, written as RegionDiff, among them RegionDiff $=\mathrm{C} 1-\mathrm{C} 2$.

\subsection{Edge Detection with Accuracy of Sub-pixel Based on Canny Operator}

The first step is to extract edge with the accuracy of subpixel from interested annular region by the operator edges_sub_pix( ). Next is to segment the edge by the operator segment_contours_xld( ). Then we adopt the operators, select_obj and get_contour_global_attrib_xld, to select and determine whether fitting circle according to the features, among them the parameter Attrib is equal to -1 means line, the parameter Attrib is equal to 0 means elliptic, the parameter Attrib is equal to 1 means round. The last step is to fit the edge contours with Tukey weighting function, by the 
operators fit_circle_contour_xld( ), gen_circle_contour_xld( ).

\subsection{Characteristic Extraction}

The first step is to read each of the separated contours and then numbered them. Finally, we adopt the operator area_center_xld( ) to calculate the area, abscissa of center and ordinate of center.

Table 1 Detection Results for Charging Hole (200mm Object Distance, Bright Light)

\begin{tabular}{|c|c|c|c|c|c|c|c|}
\hline & $\mathrm{C} 1$ & $\mathrm{C} 2$ & C3 & $\mathrm{C} 4$ & $\mathrm{C} 5$ & C6 & C7 \\
\hline Area & 1165.59 & 1216.18 & 5541.84 & 5528.41 & 5534.28 & 5514.63 & 5552.38 \\
\hline Row & 318.11 & 351.606 & 412.633 & 448.0 & 482.452 & 625.768 & 659.28 \\
\hline Column & 488.169 & 685.189 & 364.99 & 559.0 & 772.549 & 471.795 & 689.705 \\
\hline & $\mathrm{C} 1$ & $\mathrm{C} 2$ & $\mathrm{C} 3$ & $\mathrm{C} 4$ & $\mathrm{C} 5$ & $\mathrm{C} 6$ & C7 \\
\hline Area & 1180.56 & 1282.949 & 5525.0 & 5601.25 & 5577.23 & 5547.91 & 5531.28 \\
\hline Row & 315.545 & 349.279 & 436.606 & 471.321 & 506.75 & 630.079 & 665.392 \\
\hline Column & 472.003 & 669.204 & 351.003 & 524.712 & 745.031 & 419.758 & 618.501 \\
\hline
\end{tabular}

\section{Conclusion}

In this paper, it is based on vision software HALCON for platform to extract the characteristic parameters of electric vehicle charging hole, through the double judgment to detect the interested region. The first step is to carry the filtering on the original image collected. Then it adopts many image processing method, such as the modified two-dimensional image threshold segmentation based on HSI color model and the edge detection based on mathematical morphology and Canny operator. Finally, we can calculate the characteristic parameters of targets. The experimental results show that the algorithm designed can effectively detect and locate the charging hole position with accuracy rate of $100 \%$. This kind of method, compared with the existing artificial methods, has a high accuracy, high speed and good repeatability etc, which can meet the demand for automatic, fast, stable and reliable operation in the existing electric automobile industry.

\section{Acknowledgements}

The research was supported by the National Natural Science Foundation of China(NO.61401046, 61305019), National Science and Technology Support Project(NO.2015BAF11B01),Natural Science Foundation Project in Hunan Province (NO.13JJ4058), Project Supported by Scientific Research Fund of Hunan Provincial Education Department(NO.13B135), Hunan Province Key Laboratory of Videometric and Vision Navigation(TXCL-KF2013-001),Changsha Science and Technology Plan Project (K1404019-11).

\section{References}

[1] Baojun Tang, Jiangpeng Liu. Development prospect of new energy automotive industry in China[J]. Journal of Beijing institute of technology (social science edition), 2015, 11:1-6.

[2] Zhiyuan Shen,Yida Ye, Jin Wang, Guangsheng Liang, Daping zhao. Comparison and design of electric vehicle charging mode[J]. Electric applications, 2013, S2:81-83.

[3] Peichun Song. Pure electric vehicle electromagnetic compatibility analysis and electromagnetic interference suppression[J]. Journal of automotive electrical appliances, 2011, 10:59-63.

[4] G. Gilboa, N. Sochen, and Y. Zeevi. Image enhancement and denoising by complex diffusion processes. IEEE TPAMI, 2004,26(8):1020-1036. 
[5] Jian Chen, Shaohua Zheng, Lun Yu, Lin Pan. The multiple threshold adaptive median filter algorithm based on direction[J]. Journal of electronic measurement and instrument, 2013, 11:156-161.

[6] Raof, R.A.A.; Mashor, M.Y.; Ahmad, R.B.; Noor, S.S.M.; Osman, M.K., "Comparison of colour thresholding method using RGB and HSI information for Ziehl-Neelsen sputum slide images," in Information Sciences Signal Processing and their Applications (ISSPA), 2010 10th International Conference on , vol., no., pp.724-727, 10-13 May 2010.

[7] Jianjun Yin, Xinzhong Wang, Hanping MAO, Shuren Chen, Jixian Zhang. Comparison of the tomato image segmentation under the RGB and HSI color space[J]. Journal of agricultural mechanization research, 2006, 11:171-174.

[8] Qian Xu; Chakrabarti, C.; Karam, L.J., "A distributed Canny edge detector and its implementation on FPGA," in Digital Signal Processing Workshop and IEEE Signal Processing Education Workshop (DSP/SPE), 2011 IEEE , vol., no., pp.500-505, 4-7 Jan. 2011

[9] Li Baozhang; Yanping Cui, "Edge subpixel location of ellipse in computer vision measurement," 2011 3rd International Conference on Advanced Computer Control (ICACC), , vol., no., pp.452-456, 18-20 Jan. 2011

[10]Feng Wang, Guangxin Qiu, Xiaojun Cheng. The improved robust iterative least-squares plane fitting method [J]. Journal of tongji university (natural science edition), 2011,09:1350-1354.

[11]Hui Zhang, Yaonan Wang, Chengzhong Wu, Bowen Zhou, Tiejian Chen. High speed medical infusion automatic production lines visual detection and recognition technology[J]. Control theory and applications, 2014, 10, 1404-1413.

[12]Yifeng Chen. Halcon application in two-dimensional workpiece size detection[J]. Mechanical and electrical technology, 2011, 2011:12-13+17. 